\title{
A WITHIN-BURST ADAPTIVE MLSE RECEIVER FOR MOBILE TDMA CELLULAR SYSTEMS
}

\author{
E. Del Re, G. Castellini", L. Pierucci, F. Conti \\ Universita' di Firenze \\ Dipartimento di Ingegneria Elettronica \\ Via Santa Marta, 350139 FIRENZE ITALY \\ * I.R.O.E.- CNR \\ Via Panciatichi, 6450127 FIRENZE ITALY
}

\begin{abstract}
The paper describes a simplified structure concerning the implementation of an adaptive digital receiver suitable for the pan-european cellular system (GSM Group Special Mobile).

In the GSM system, the performance of the received information is strongly affected by the multipath typical of the mobile communications channel.

The proposed maximum likelihood sequence estimation (MLSE) receiver based on the Viterbi algorithm compensates for selective distortion due to the multipath propagation and Doppler shift also in the case of fast channel variations using an adaptive tracking mode method.
\end{abstract}

\section{INTRODUCTION}

The growing demand of mobile radio telephone service has accelerated the development of a new high-capacity pan-European digital mobile radio system,i.e. the ETSI/GSM (European Telecommunications Standards Institute/Group Special Mobiles).

This system employs time division multiple access (TDMA) with eight channels per carrier with GMSK (Gaussian Minimum Shift Keying) modulation and a burst transmission with modulating data signal rate equal to $270.833 \mathrm{~kb} / \mathrm{s}$.

The modulation (of the partial response type) and the intersymbol interference resulting from the time-variant multipath propagation of the signal through the channel the requires a maximum likelihood sequence estimation (MLSE). An efficient implementation of the MLSE criterion is the Viterbi algorithm (VA) for demodulation.

The VA requires the knowledge of the channel. The channel can be estimated using a training sequence, positioned in the middle of the burst and this estimate can be used for the entire burst.

However, the rapidly varying channel characteristics within the burst period, due to fading and to the vehicle speed may require a more efficient design of the adaptive receiver.

The paper first presents the general receiver characteristics including the performance and the implementation complexity.

The performance of the receiver is evaluated using a channel simulator suitable for mobile communications. The simulated channel impairments are:

- flat Gaussian noise (as a reference case)

- Rayleigh (or Rice) fading with Doppler frequency shift and multiple echoes selected by the GSM Propagation Group as representative of urban area(TU), rural area (RA) [1].

The paper also shows the performance improvement obtained by using adaptation algorithm (LMS) to the MLSE receiver when the tracking of rapidly timevarying distortions is needed.

\section{DIGITAL RECEIVER ARCHITECTURE}

The receiver is specifically tailored for the applications with GSMK modulation schemes and modulation index $\mathrm{h}=\mathbf{0 . 5}$.

The general structure of the adaptive digital receiver is shown in Fig. 1.

A discrete-time equivalent model of the overall channel characterizes the system that precedes the Viterbi receiver. Including the noise component, the received signal results

$$
\begin{gathered}
y(n T)=\sum_{k} h_{n}(k T) a_{n-k}+w(n T)= \\
=s(n T)+w(n T)
\end{gathered}
$$

where

- $\quad a_{n}= \pm 1$ are the transmitted data symbols

- $\quad h_{n}(k T)$ is the complex time-varying lowpass

IV $\mathbf{- 4 9 3}$ 


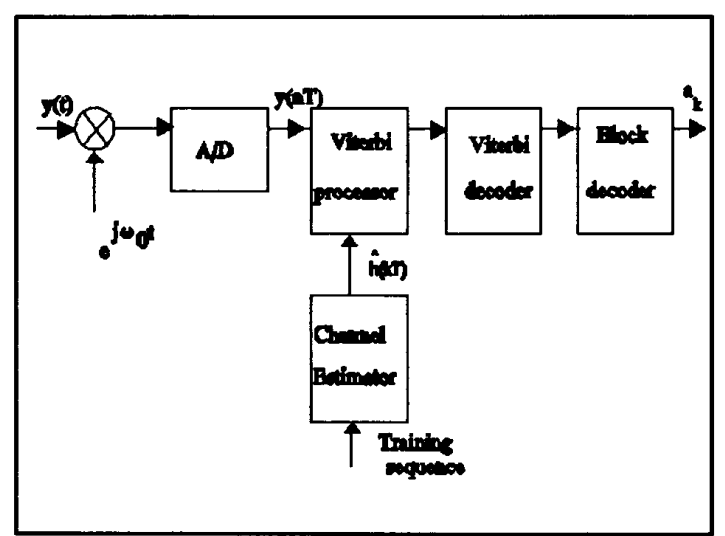

Figure 1 - General receiver structure for the GSM system

equivalent impulse response of the all transmission system including the pulse shape modulation filter, the channel and the receiving filter

- $\quad w(n T)$ are the samples of an additive noise term.

For additive independent Gaussian noise components, it is known that the MLSE criterion leads to a receiver that has to select among all possible data vectors the vector $\hat{A}$ whose corresponding signal vector $\hat{\mathbf{S}}$ is closed in Euclidean distance to the observation vector Y. In other words it selects the vector Â such that minimizes

$$
\|Y-\hat{S}\|^{2}=\sum_{i=1}^{M}|y(i T)-\hat{s}(i T)|^{2}
$$

The implementation of the MLSE criterion with the above metric can be efficiently performed by the wellknown Viterbi algorithm. From the computational point of view, the Viterbi algorithm applied to the MLSE requires the evaluation of the values of the signal component $s(\mathrm{iT})$ for all the possible combinations of data symbols, in order to update the Euclidean distance. At the end of a block of $M$ received samples, the Viterbi algorithm determines the $M$ data symbols that minimizes (2), that represents the MLSE of the transmitted data symbols.

It is important to point out that the Viterbi algorithm:

i) requires the knowledge or the estimate of the equivalent channel impulse response $h_{n}(k T)$. In the GSM system, the data packet contains a pseudorandom sequence of 26 bits, termed midamble, which is known at the receiver. By using the correlation properties of this sequence an estimate of the communication channel during the actual time-slot can be performed . $h_{n}(k T)$ must be of the FIR type, say of order $N$ $(\mathrm{N}=5), \mathrm{k}=0,1, \ldots \mathrm{N}-1$;

The MLSE adaptive receiver consists essentially of two blocks: the channel estimator and the Viterbi processor as shown in Fig. 2.

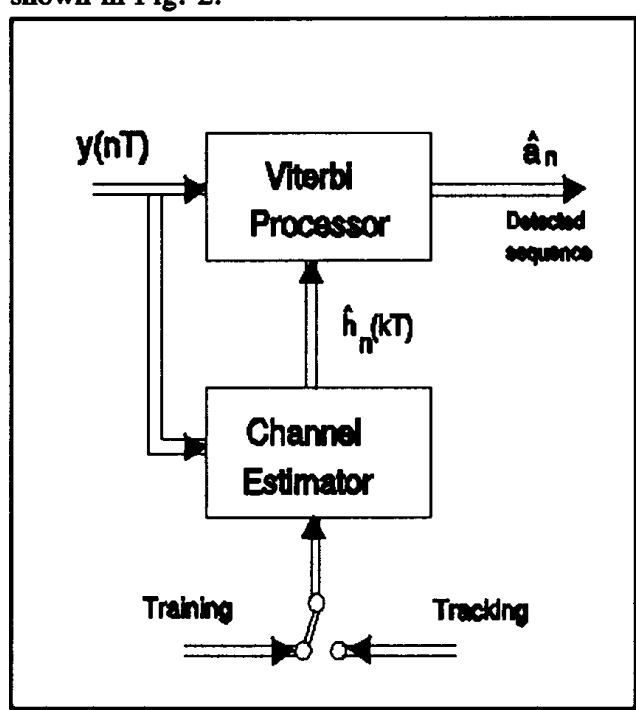

Figure 2 - Block diagram of MLSE adaptive receiver

Furthermore other considerations are important from the implementation point of view of the digital receiver.

A) The preamble position in middle of the burst allows to produce a channel impulse response estimate that in many situation is sufficiently accurate for the whole duration of the burst even in presence of time-varying multipath fading. For this reason the Viterbi algorithm is initialized at the extremes of the midamble and then works on the informative sequence in direct and inverse propagation. As expected the performance of the receiver is worse at the beginning and at the end of the burst, and in the case of high speed of the mobile it is necessary to update the fast channel response variations by a receiver adaptability (tracking mode as shown in Fig.2) using LMS algorithm.

B) As long as the equivalent baseband 
representation of the communication channel is correct, the MLSE receiver only needs the estimate of the channel impulse response $h_{n}(k T)$ and does not require the knowledge of the carrier phase and of the symbol timing. These information are included automatically in the channel estimate $\hat{h}_{n}(k T)$. Therefore the MLSE receiver does not require subsystems dedicated to the carrier phase and symbol timing extraction. Moreover even a moderate frequency offset between the carrier frequency and the frequency of the receiver local carrier can be tolerated. The frequency offset appears as a slowly time-varying relative carrier phase that contributes to the time variations of the channel impulse response. These variations can be tracked by adaptation algorithms.

C)

The value of the parameter $\mathbf{N}$ affects the receiver complexity. However, in practice the proposed receiver is robust with respect to the value of $N$. If turns out that the receiver performance (in terms of error probability) is quite good even for values of $\mathrm{N}$ significantly smaller than the actual duration of the channel impulse response. Moreover it turns out that the digital implementation of the MLSE receiver requires short binary register for a satisfactory performance, i.e. the receiver is robust with respect to a finite-arithmetic implementation.

The characteristics outlined above suggest that the presented MLSE receiver is a good candidate for a digital receiver for mobile communications.

\section{MLSE RECEIVER WITH LMS CHANNEL ADAPTIVITY}

In the GSM system very fast adaptation methods are necessary to compensate for the time and the frequency selective distortion of the fading channel varying very rapidly with respect to the bit rate. This is particular evident in the case of high vehicle speed of the order of $300 \mathrm{~km} / \mathrm{h}$ or more.

In the case of the proposed MLSE receiver the midamble of 26 bits is used to estimate the communications channel during the actual burst (training mode).

The channels model can be approximated as a linear FIR filter with adjustable coefficients and by the correlation method the short known sequence gives an initial estimate of the coefficients for each bursts.

This estimate can be held fixed during the burst and is updated only the next burst.

When the channel characteristics vary very rapidly it is necessary to update the channel estimate within the burst (tracking mode).

The most commonly used criterion to optimize the channel coefficients is the minimization of the mean square error (MSE) between the desired data signal and the actual signal samples. The error is defined as

$$
\begin{gathered}
e(n)=y(n T)-\sum_{k} h_{n}(k T) \hat{a}_{(n-k)} \\
=y(n T)-\hat{s}(n T)
\end{gathered}
$$

The minimization of the MSE carries to the Wiener Hopf solution where the computing of autocorrelation matrix inverse of the vector of the estimated data signal samples is needed.

The calculation is expensive and the estimate $\mathbf{s}(\mathbf{n T})$ can be obtained if the data sequence is known.

Alternatively, the minimization of the mean square error may be obtained recursively using the LMS algorithm by Widrow

$$
\begin{gathered}
h_{(n+1)}(k T)=h_{n}(k)+\Delta e_{n} y^{*}(n-k) T \\
k=0,1 \ldots N-1, \forall n
\end{gathered}
$$

where $h_{n}(k T)$ is the vector of the channel coefficients at the instant $\mathrm{nT}, \mathrm{y}(\mathrm{nT})$ is the received signal samples (* indicates the complex conjugate), $e_{n}$ is the error signal between the desired signal and the estimated signal and $\Delta$ is the step size selected as a compromise between the speed of convergence and the stability.

This adaptation algorithms try to improve the channel estimate supplied to the Viterbi demodulator. In the literature, it is common to use this estimated data sequence at the output of the Viterbi demodulator as the "true" sequence to estimate $\hat{\mathbf{s}}(\mathrm{nT})$ and update the channel coefficients. However, in this approach it is unavoidable a delay to obtain the data sequence recognized by the VA demodulation and to feed back this sequence in the update of $\hat{\mathbf{s}}(\mathrm{nT})$. Alternatively, the estimate can be evaluated by using the data sequence of a particular survivor in the branch metric calculation during the Viterbi trellis. The selected data sequence in our case corresponds to the minimum state metric at time $\mathrm{nT}$, and it is used to update the estimate $\mathrm{s}(\mathrm{nT})$ of all the survivors. This local estimate is therefore updated within the burst during the entire information sequence. 


\section{RESULTS AND CONCLUSIONS}

The following figures compare the performance of the digital MLSE receiver that uses the channel estimate obtained by the midamble training sequence for the whole burst (A) with the receiver using an adaptive tracking algorithm. Curve $B$ represents the performance of the adaptive receiver within the burst implemented by the LMS algorithm as in eq. (4), with $\Delta=0.01$ in RA case and $\Delta=0.03$ in TU case. Curve $C$ shows the performance of the theoretically optimum adaptive receiver (Wiener-Hopf solution) based on the knowledge of the channel impulse response during the whole burst. Of course, this performance represents a lower bound for any realizable receiver as this knowledge is not available and an estimate must be obtained.

As shown, the necessity of an adaptive algorithms is more evident in the case of high vehicle speed, as it happens in the rural area where the adaptation algorithms can recover the error due to the Doppler shift.

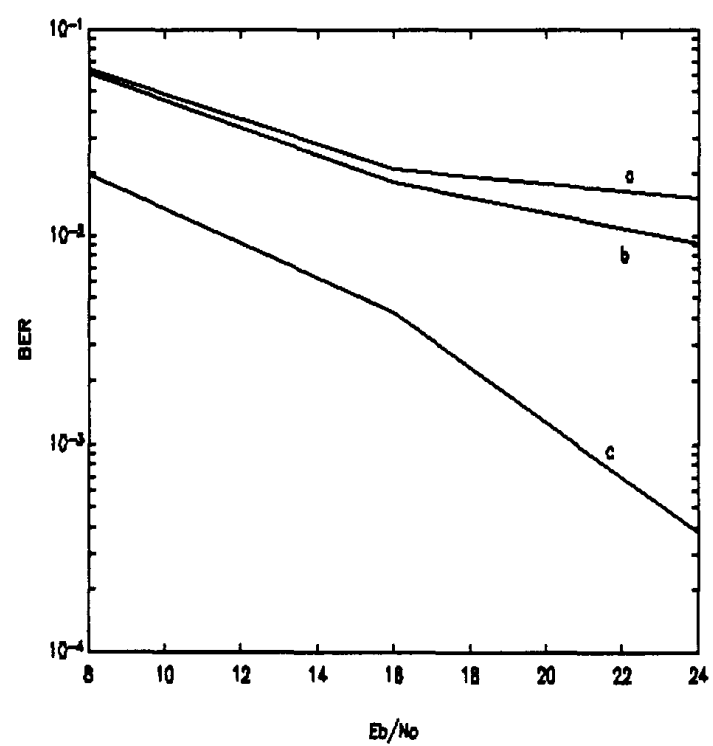

Figure $3-R A$ case with vehicle speed of 250 $\mathrm{km} / \mathrm{h}$

The results highlight the slowly convergence rate of the LMS algorithms due to the fact that only one

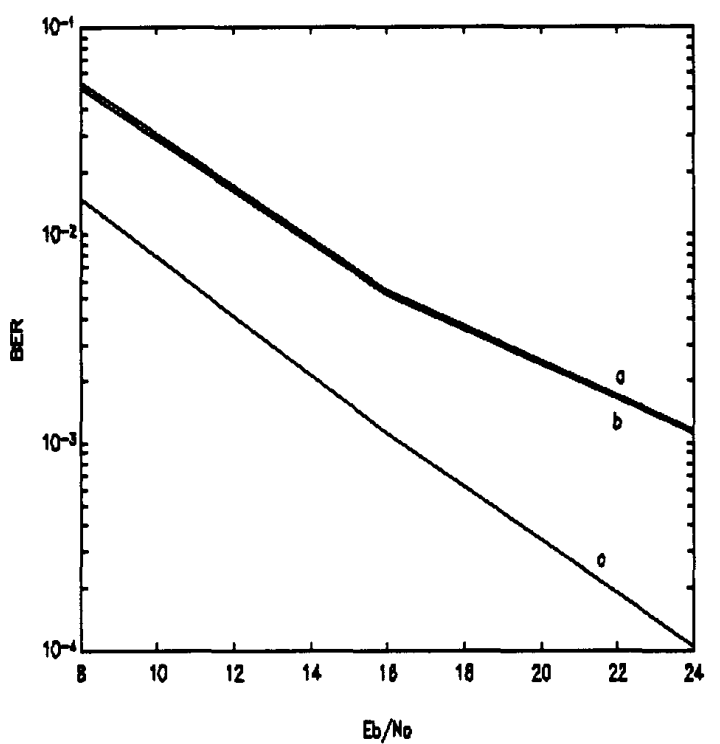

Figure 4 - TU case with vehicle speed $50 \mathrm{~km} / \mathrm{h}$

parameters $\Delta$ controls the adaptation rate. Therefore to approach the good results of the Wiener-Hopf solution the use of different adaptive algorithm (e.g. of the fast Kalman-type) should be used. In general these algorithms achieve faster convergence and tracking capabilities even though they are more complex to implement.

\section{REFERENCES}

[1] CEPT/CCH/GSM Recommendations, Series 05.

[2] G.J.Saulnier, C.Mcd.Puckette,IV, R. C. Gaus,Jr., R.J. Dunki-Jacobs, T.E. Thiel "A VLSI demodulator for digital RF network applications: Theory and Results", IEEE Journal on Selected Areas in Communications, Vol. 8, N.8, Oct. 1990.

[3] R. D'Avella, L. Moreno, M. Sant'Agostino, "An adaptive MLSE receiver for TDMA digital mobile radio", IEEE, J. of Selected Areas on Communications, vol. 7, $\mathrm{N}^{\circ} 1$, p.122-129, January 1989.

[4] E.Del Re, G.Castellini, R.Fantacci, G.Benelli, L.Pierucci, L.Pogliani, "Design of a digital MLSE receiver for Mobile Radio Communications" Globecom 91 Phoenix 